Curriculum Parodontologie

1. Termin: 1.-2.2.13

Moderator: Prof. S. Jepsen, Bonn

Kurs-Nr.: $\quad 0419.3$

Strukturierte Fortbildung:

Funktionsanalyse und -the-

rapie für die tägliche Praxis

Hands-On-Kurs

$\begin{array}{ll}\text { 1. Termin: } & \text { 8.-9.3.13 } \\ \text { Referent: } & \begin{array}{l}\text { Dr. U. Harth, } \\ \text { Bad Salzuflen }\end{array} \\ \text { Kurs-Nr.: } & 1001.9\end{array}$

Krieg oder Frieden - Vom

Umgang mit Versicherun-

gen und Beihilfestellen

$\begin{array}{ll}\text { Termin: } & 16.3 .13 \\ \text { Referent: } & \begin{array}{l}\text { Dr. M. Cramer, } \\ \text { Overath }\end{array} \\ \text { Gebühr: } & 175,-€ \\ \text { Kurs-Nr.: } & 5038.3\end{array}$

Curriculum Kinder- und JugendzahnMedizin

$\begin{array}{ll}\text { 1. Termin: } & \text { 22.-23.3.13 } \\ \text { Moderator: } & \text { Prof. C. H. Splieth, } \\ & \text { Greifswald } \\ \text { Kurs-Nr.: } & 4020.7\end{array}$

Curriculum für Hypnose und Kommunikation in der Zahnmedizin

1. Termin: 5.-6.4.13

Moderator: Dr. H. Freigang, Berlin Kurs-Nr.: $\quad 6030.5$

\section{MKG-Update 2013}

25. / 26. Januar 2013 in Wiesbaden Wissenschaftliche Leitung: Knut A. Grötz, Wiesbaden; Andrea M. Schmidt-Westhausen, Berlin; Wilfried Wagner, Mainz Seminarort:

Kurhaus Kolonnaden Wiesbaden, Kurhausplatz 1, 65189 Wiesbaden Teilnahmegebühren:

reduziert* $375,-€$, regulär $400,-€$ Ermäßigung:

Assistenzarzt** $-25,-€$, alle

Gebühren inklusive gesetzlicher

Mehrwertsteuer;

* die reduzierte Gebühr erhalten Sie bis 1 Woche vor Veranstaltungsbeginn; danach erhöhen sich die Teilnahmegebühren um 25,- $€$ (reguläre Gebühr).

** Nachweis als Assistenzarzt erforderlich

\section{Anmeldung}

wikonect GmbH Wiesbaden

Fax: 0611/204809-10

Tel.: 0611/204809-19

Internet: http://www.mkg-update.com

Konzept \& Veranstalter:

med update $\mathrm{GmbH}$ Wiesbaden

www.med-update.com
Curriculum

Ästhetische Zahnmedizin

1. Termin: 3.-4.5.13

Moderator: Prof. Roland Franken-

berger, Marburg 4044.3

Curriculum Endodontie

\section{Termin: 24.-25.5.13 \\ Moderator: Prof. Michael Hüls- \\ Kurs-Nr.: $\quad 4036.5$}

Strukturierte Fortbildung: Akupunktur für Zahnärzte
1. Termin:
14.-15.6.13
Referent:
Dr. J. Gleditsch, Wien
Kurs-Nr.:
6081.0

Strukturierte

Fortbildung: Chirurgie

$\begin{array}{ll}\text { 1. Termin: } & \text { 25.-26.10.13 } \\ \text { Referent: } & \text { Prof. A. Filippi, Basel } \\ \text { Kurs-Nr.: } & 0603.4\end{array}$

8. Internationales

Wintersymposium der Deutschen Gesellschaft für Orale Implantologie

24. Februar bis 1. März 2013 in Zürs am Arlberg

Update Implantologie 2013: Standards und Workflows in der Übersicht

Um wissenschaftlich fundierte Standards und Arbeitsabläufe für den Praktiker geht es beim 8 . Internationalen Wintersymposium der Deutschen Gesellschaft für Orale Implantologie (DGOI). Prof. Georg-H. Nentwig, Fortbildungsreferent der DGOI, stellt unter dem Leitthema „Implantologisches Update 2013“" ein exzellentes Programm mit namhaften Referenten zusammen. Beleuchtet werden unter anderem verschiedene Aspekte der Bereiche Knochenregeneration, Weichgewebsmanagement, Sofortversorgung und der periimplantären Infektion. Die 1-wöchige Fortbildung findet im Robinson Club Alpenrose in Zürs am Arlberg statt. Die Infrastruktur und die vielseitigen Sportmöglichkeiten bieten auch den mitreisenden Partner und Familien viel Abwechslung. Zum Rahmenprogramm gehören das Slalom-Skirennen und der zünftige Hüttenabend am Mittwoch.

Informationen und Anmeldung DGOI Büro

Bruchsaler Straße 8

76703 Kraichtal

Tel.: 07251/618996-0

Fax: 07251/618996-26

E-Mail: mail@dgoi.info

Internet: www.dgoi.info
Aufstiegsfortbildung zum/r Dentalhygieniker/in

Seminar- ZÄ I. Kronfeld-

leiterin: Möhring

Kurszeit: $\quad$ April 2013-April 2014

Gebühr: $\quad$ 14850,00€

Kurs-Nr: $\quad 0808.0-$

\section{Information und Anmeldung}

Philipp-Pfaff-Institut Fortbildungseinrichtung der Zahnärztekammer Berlin und Landeszahnärztekammer Brandenburg

Ansprechpartnerin für Fortbildungskurse: Nadine Krause, Aßmannshauser Str. 4-6, 14197 Berlin, Tel.: 030/414725-40, Fax: 030/4148967,

E-Mail: info@pfaff-berlin.de, Internet: www.pfaff-berlin.de

Ästhetische Versorgungen mit VITABLOCS RealLife und VITA Rapid Layer Technologie

Termin: 7.-8.12.12

Ort: Geilenkirchen

Referent: $\quad$ ZTM Wolfgang

Sokalla

Mehr Effizienz und Erfolg mit dem CEREC / inLab System

Termin: $\quad$ 5.2.6.2.13

Ort: Leipzig

Termin: $\quad$ 20.2.-21.2.13

Ort: Schweig

Referentin: Marianne Höfermann

Gebühr: $\quad 580,-€$

CAD/CAM-Restaurationen im Frontzahnbereich

Termin : $\quad$ 7.2.-8.2.13

Ort: $\quad$ Leipzig

Termin: 25.2.-26.2.13

Ort: $\quad$ Schweig

Referentin: Marianne Höfermann

Gebühr: $\quad 620,-€$

Information und Anmeldung

Ute Schmidt, Tel.: 07761/562-235,

E-Mail: u.schmidt@vita-zahnfabrik.com

59. Zahnärztetag der Zahnärztekammer Westfalen-Lippe

6.-9. März 2013 in Gütersloh Thema: PAR-Therapie heute: Was ist das Beste für meine Patienten? Tagungspräsident: Prof. Dr. Hannes Wachtel, München

Zahnärztekammer Westfalen-Lippe Akademie für Fortbildung Auf der Horst 31, 48147 Münster Tel.: 0251/507627

Fax: 0251/50765627

E-Mail: annika.bruemmer@ zahnaerzte-wl.de 